\title{
CNN-Based Spectral Super-Resolution of Panchromatic Night-Time Light Imagery: City-Size-Associated Neighborhood Effects
}

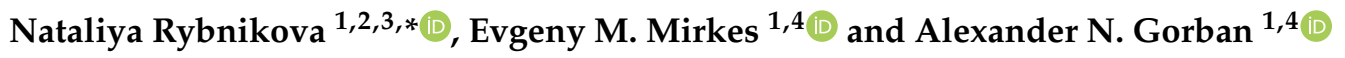 \\ 1 Department of Mathematics, University of Leicester, Leicester LE1 7RH, UK; em322@leicester.ac.uk (E.M.M.); \\ a.n.gorban@leicester.ac.uk (A.N.G.) \\ 2 Department of Natural Resources and Environmental Management, University of Haifa, Haifa 3498838, Israel \\ 3 Department of Geography and Environmental Studies, University of Haifa, Haifa 3498838, Israel \\ 4 Institute of Information Technologies, Mathematics, and Mechanics, Lobachevsky University, \\ 603105 Nizhny Novgorod, Russia \\ * Correspondence: nataliya.rybnikova@gmail.com; Tel.: +972-54-55-147-55
}

Citation: Rybnikova, N.; Mirkes, E.M.; Gorban, A.N. CNN-Based Spectral Super-Resolution of Panchromatic Night-Time Light Imagery: City-Size-Associated Neighborhood Effects. Sensors 2021, 21,7662. https://doi.org/10.3390/ s21227662

Academic Editor: Andrzej Stateczny

Received: 2 October 2021

Accepted: 16 November 2021

Published: 18 November 2021

Publisher's Note: MDPI stays neutral with regard to jurisdictional claims in published maps and institutional affiliations.

Copyright: (c) 2021 by the authors. Licensee MDPI, Basel, Switzerland. This article is an open access article distributed under the terms and conditions of the Creative Commons Attribution (CC BY) license (https:/ / creativecommons.org/licenses/by/ $4.0 /)$.

\begin{abstract}
Data on artificial night-time light (NTL), emitted from the areas, and captured by satellites, are available at a global scale in panchromatic format. In the meantime, data on spectral properties of NTL give more information for further analysis. Such data, however, are available locally or on a commercial basis only. In our recent work, we examined several machine learning techniques, such as linear regression, kernel regression, random forest, and elastic map models, to convert the panchromatic NTL images into colored ones. We compared red, green, and blue light levels for eight geographical areas all over the world with panchromatic light intensities and characteristics of built-up extent from spatially corresponding pixels and their nearest neighbors. In the meantime, information from more distant neighboring pixels might improve the predictive power of models. In the present study, we explore this neighborhood effect using convolutional neural networks (CNN). The main outcome of our analysis is that the neighborhood effect goes in line with the geographical extent of metropolitan areas under analysis: For smaller areas, optimal input image size is smaller than for bigger ones. At that, for relatively large cities, the optimal input image size tends to differ for different colors, being on average higher for red and lower for blue lights. Compared to other machine learning techniques, CNN models emerged comparable in terms of Pearson's correlation but showed performed better in terms of WMSE, especially for testing datasets.
\end{abstract}

Keywords: night-time light (NTL); panchromatic; red; green; blue (RGB) bands; international space station (ISS); convolutional neural network (CNN); neighborhood effect

\section{Introduction}

Artificial night-time lights (NTL), emitted from the residential, industrial, and entertainment areas, and captured by satellites, provides researchers and policy-makers with the information for a wide range of analyses: on the human presence on the Earth [1-8], on NTL adverse effects on human health [9-11], on the health of ecosystems [12,13], on night sky observations [14-16], etc. Globally, this NTL information is currently provided by the day-night band (DNB) sensor, supported by Visible Infrared Imaging Radiometer Suite (VIIRS), and available from the Earth Observation Group site [17]. These data, however, are panchromatic (that is, each pixel of NTL image reports summarized intensities of light in the diapason of 500-900 nm [18]). In the meantime, information about NTL color is of great importance for a variety of research, since it is known, for instance, that NTL emissions of different diapasons are associated with different economic activities and land-use types [19-21], or that NTL in blue diapason is especially effective in melatonin suppression [22] and thus inducing hormone-dependent cancers [23] and obesity [24]. Red, 
green, and blue (RGB) spectrum of NTL data, however, are currently available from the International Space Station (ISS) for selected areas and sporadically [25], or on a commercial basis [26], thus impeding research in a variety of fields. The present study proposes an approach to obtain RGB NTL information, highly demanded the epidemiological and nature conservation research from freely available panchromatic NTL and auxiliary built-up area data.

In our previous research [27], we attempted to obtain RGB NTL estimates from freely available panchromatic VIIRS-DNB NTL data and information on built-up areas, used as land-use type proxies. We used several machine learning techniques, such as linear regressions, non-linear kernel regression, random forest, and elastic map models to define the association between ISS-provided RGB NTL levels at certain pixels and a set of predictors, comprising panchromatic NTL and built-up areas characteristics from corresponding pixel and its first-order neighbors. We built such models for each of the eight metropolitan areas all over the world-Atlanta (US), Beijing (China), Haifa (Israel), Khabarovsk (Russia), London (UK), Naples (Italy), Nashville (US), and Tianjin (China) -and validated them over the rest seven metropolitan areas. The analysis showed that model-estimated RGB NTL levels demonstrated a sufficient consistency with the original ISS-provided RGB NTL data: Pearson's correlation coefficients, both for training and testing sets, ranging between 0.719 and 0.963 , and weighted mean squared error (WMSE) varying from 0.029 to 4.223 . This study considered the first-order neighbors of each point only. In the meantime, accounting for the information from more distant neighboring pixels (than first-order ones) might be beneficial for obtaining more accurate estimates of RGB NTL levels of the pixel under analysis. The reason is that certain land-use types within a city (residential areas, industrial facilities, municipal roads) may differ in physical size, NTL intensity, and spectral characteristics [19-21]. In the present study, we aim at assessing this neighborhood effect.

Since we aim at enhancing the spectral resolution of NTL images from panchromatic to $\mathrm{RGB}$, our task might be viewed as a super-resolution (SR) task, which refers to the process of recovering high-resolution images from low-resolution ones [28]. SR, which initially referred to the spatial aspect of the images only, is nowadays one of the most active research areas [29]. Spatial SR research utilizes either frequency-domain-based, interpolationbased, regularization-based signal processing techniques, or machine-learning methods (for reviews, see [30-32]). Recently, with the development of deep learning techniques, convolutional neural networks (CNN) have widely been utilized in spatial SR research (see, for example, [33-38]; for review, see [28]). In line with spatial, spectral SR research, modeling the mapping from coarser scale spectrum (usually, RGB) to finer one (usually, hyperspectral images of over 30 spectral channels) [39], is also nowadays quickly developing. Compared to spatial, spectral SR research is much more sparse [40] mainly due to the lack and incomparability of training data: hyperspectral cameras are expensive and generally do not simultaneously produce broader RGB bands-thus, low and high spectral resolution images vary in terms of the scene. Additionally, spectral responses of sensors are camera-specific and may vary significantly from camera to camera [41]. Thus, recent studies on spectral SR utilize hyperspectral images, comprised of $\sim 30$ bands from 400 to 700 nanometers with $10 \mathrm{~nm}$ increment, from the ICVL (201 images), CAVE (32 images), and NUS (66 images) datasets (see, for example, [40-43]). Probably due to later start, spectral SR studies are often based on neural networks, either shallow (see, inter alia, [42-44]), or moderately and very deep (see, inter alia, [40,41], correspondingly) CNNs. A shallow neural network approach implies using a high-quality hyperspectral dataset to create a sparse dictionary of hyperspectral signatures and their RGB projections and afterward to restore a hyperspectral image from decomposed-over the dictionary-pixels of a new RGB image [42-44]. This approach is reported (see [43]) to be much faster in computation, but comparable in terms of performance with its predecessor, based on the matrix factorization method [45]. Simultaneously, a shallow neural network approach was reported (see [42]) to be similar in performance in terms of RMSE to very deep CNN, with a total of 56 layers [41]. The 
reason might be in the lack of training data and high correlation between spectral bands, which require a moderately deep CNN; as a recent study confirms (see [40]), such a CNN, consisting of 6 layers only, outperforms both shallow and very deep CNN.

Despite the formal proximity to the image colorization task, also-as in our case-implying enhancing the spectral resolution of the image from grayscale to RGB [46], our task is essentially different. In image colorization, mainly applied to historical photographs and videos, the research, aimed at diminishing the user input, is generally based upon finding an appropriate reference image (or selected semantic feature) from a dataset and transferring its color to the whole or the part of the image under analysis [47-52]; the research is built upon semantic features (such as cars, animals, plants, etc.) identification and allocation.

In essence-not formally-our task is different from the image colorization task. Unlike the latter, our research does not imply user intervention or semantic features identification. Actually, our task is a spectral SR task. The difference is that we deal with night-time satellite images, which, due to low signal, are not available in the hyperspectral format, and it is a great privilege to obtain even RGB resolution. Given the ill-posedness of the task of mapping between coarser and finer spectral resolution [39-41,43], in our research, we use built-up area characteristics as additional input information. We hypothesize that panchromatic light and built-up area information from neighboring pixels might contribute to better predictions of RGB levels. Using CNN as a tool, we settle our task as a regression one: we form multi-layer small-size images from panchromatic VIIRS-DNB NTL and builtup area data, which are further used as CNN input images, and match them with the levels of either red, green, or blue light pixels, located in the center of the corresponding small-size image, and used as a dependent variable. We run our analysis upon eight metropolitan areas all over the world. We experiment with the size of input CNN images-we try square images with an odd number of pixels (from $3 \times 3$ to $15 \times 15$ ). We compare the performance of the models overtraining and testing datasets for each CNN input image size and also compare the fit to the actual RGB data of the CNNs with previously used machine learning techniques [27]. As our results indicate, compared to our previous study, under-examined first-order neighborhood effect via linear regression, non-linear kernel regression, random forest, and elastic map models [26], CNN models emerged comparable in terms of Pearson's correlation, but showed generally better performance in terms of WMSE, especially for testing datasets. We assume that such an improvement was induced by accounting for the varying neighborhood effect. The second important finding of the analysis is that for relatively small metropolitan areas, either in terms of area or population, the best-performing models for any color light band prediction were built for $5 \times 5$ input image size, while for larger areas the optimal input image size was at least $7 \times 7$ pixels and varied depending on the color prediction.

\section{Materials and Methods}

\subsection{Data Sources}

In the analysis, we use three sources of information. First, as an output of CNNs, we use multispectral (RGB) images, provided by the ISS and available from the Astronaut Photography Search Photo service [25] for eight metropolitan areas all over the world-Atlanta (US), Beijing (China), Haifa (Israel), Khabarovsk (Russia), London (UK), Naples (Italy), Nashville (US), and Tianjin (China). The images and their ID numbers are reported in the left column of Figure 1.

Second, as CNN inputs, we use panchromatic NTL intensities-namely, we use spatially corresponding cropped areas from VIIRS-DNB-provided images, available from the Earth Observation Group site [17] (see the central column of Figure 1). In the present analysis, to avoid poor-quality pixels, outliers, and cloud contamination, which might be present in daily VIIRS-DNB images [53], we use their monthly composites.

Third, as additional inputs for CNNs, we use the characteristics of the built-up extent; That is, we use cropped corresponding areas from the global raster layer of human built- 
up area and settlement extent (HBASE) database, reporting the pixel-wise percentage of the built-up area in the range from 0 to $100 \%$. These data are available from the NASA Socioeconomic Data and Application Center site [54] (see right panel of Figure 1).
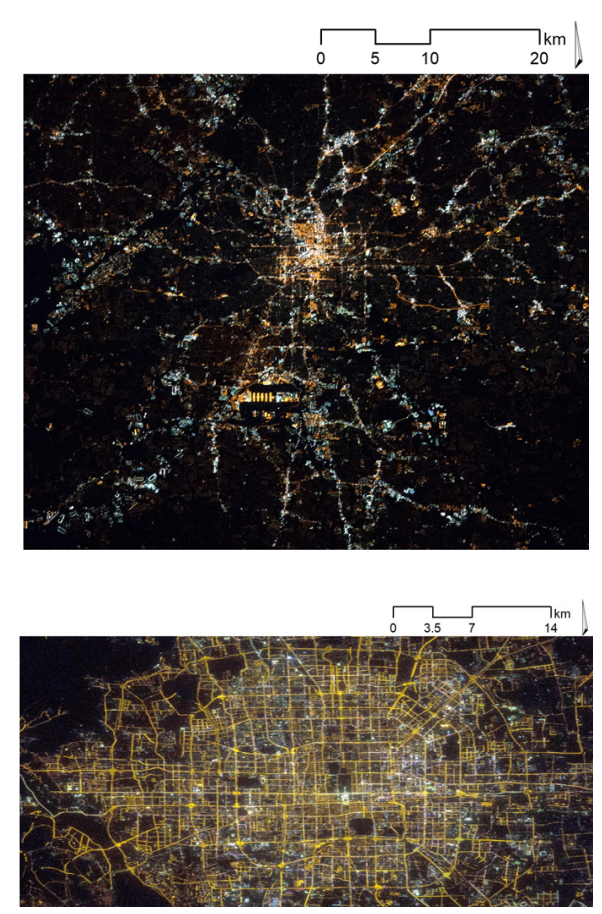

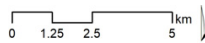

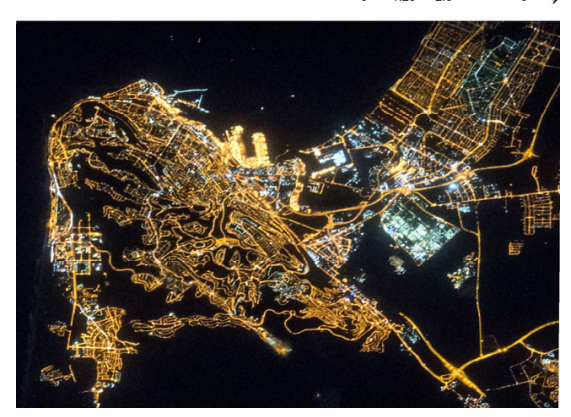

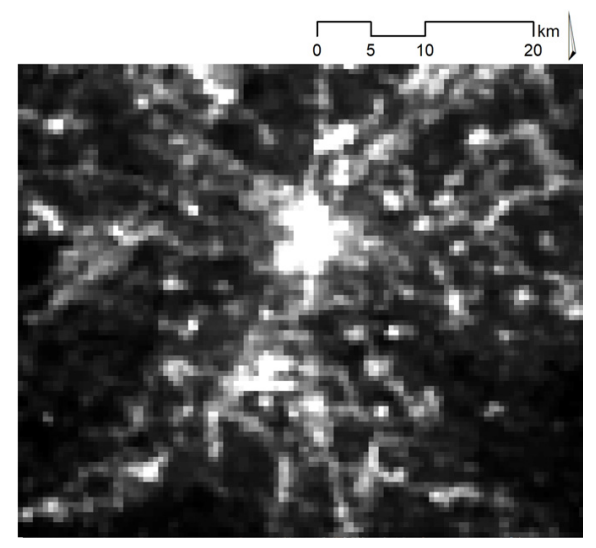

Atlanta (ISS047-E-26897)

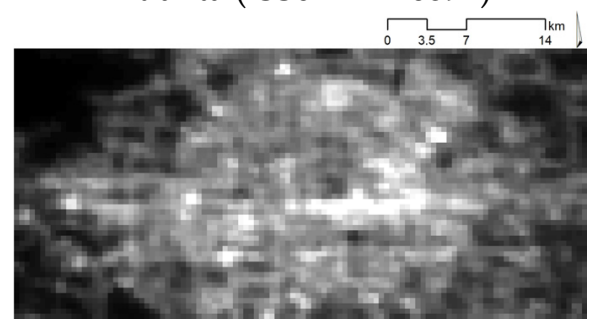

Beijing (ISS047-E-11998)

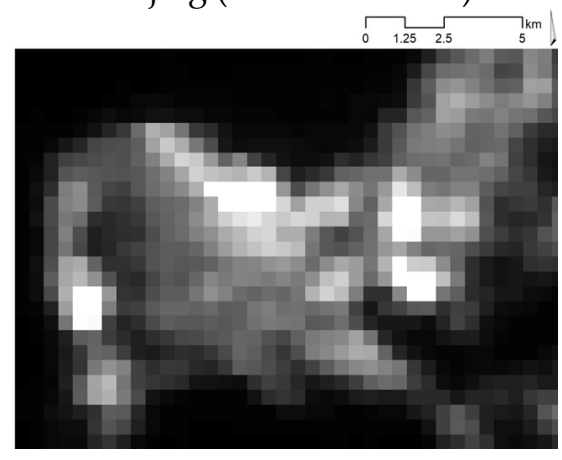

Haifa (ISS045-E-148262)
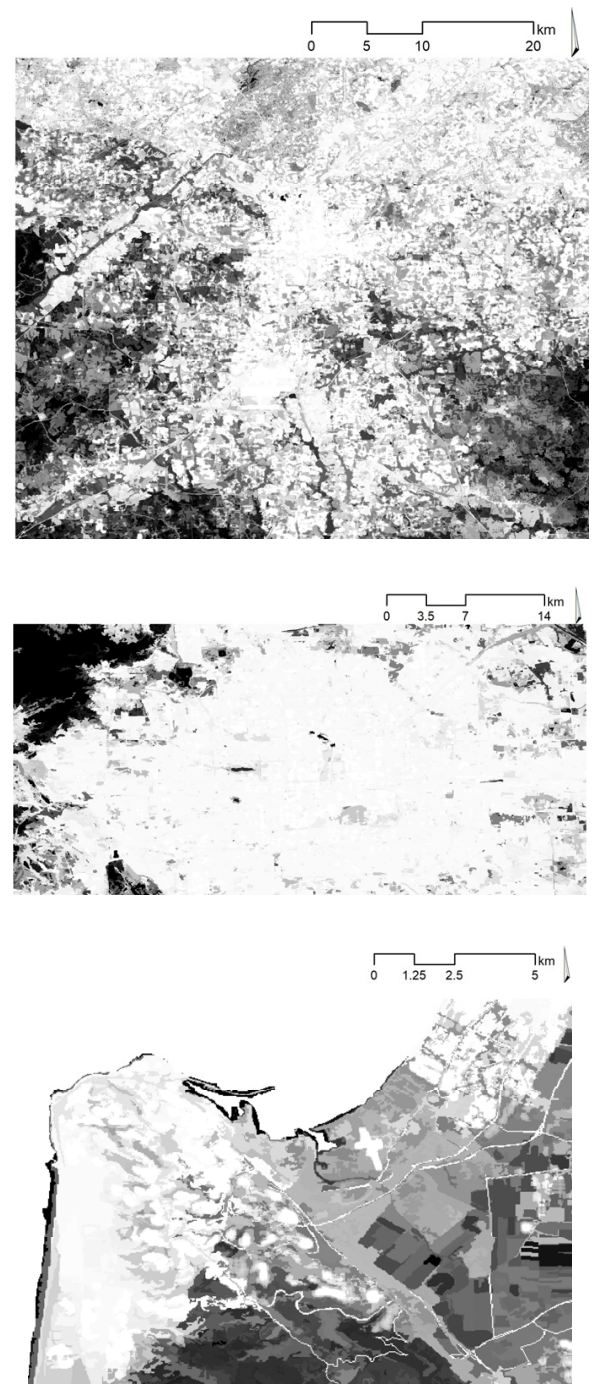

Figure 1. Cont. 

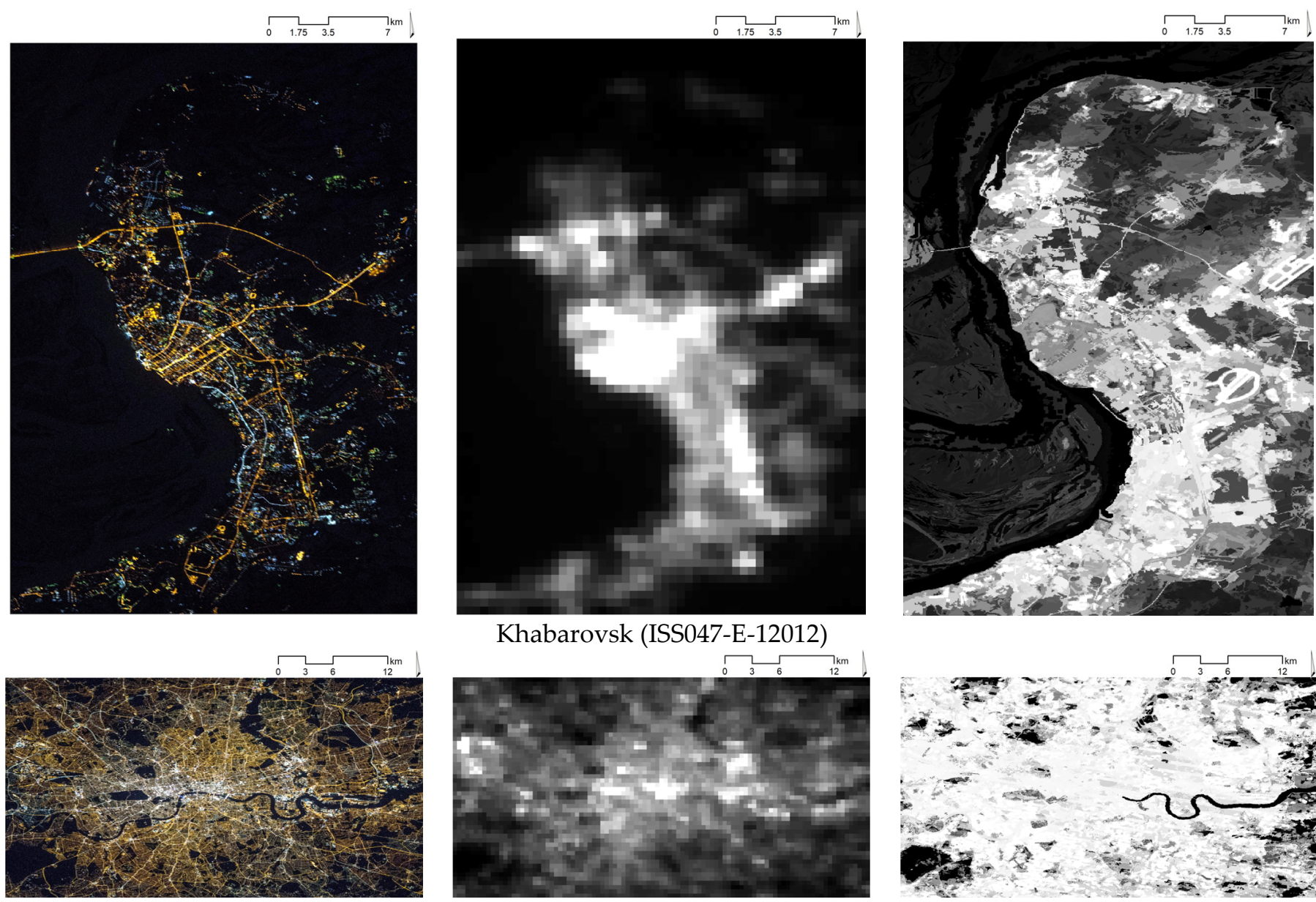

Khabarovsk (ISS047-E-12012)

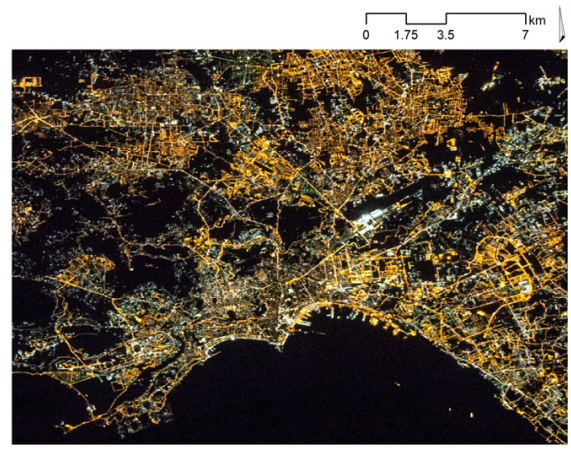

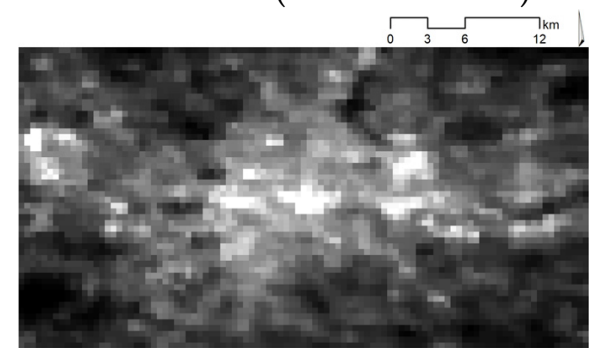

London (ISS045-E-32242)

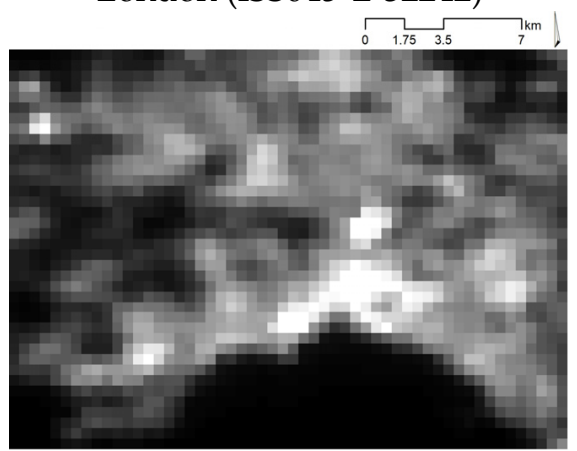

Naples (ISS050-E-37024)

Figure 1. Cont.
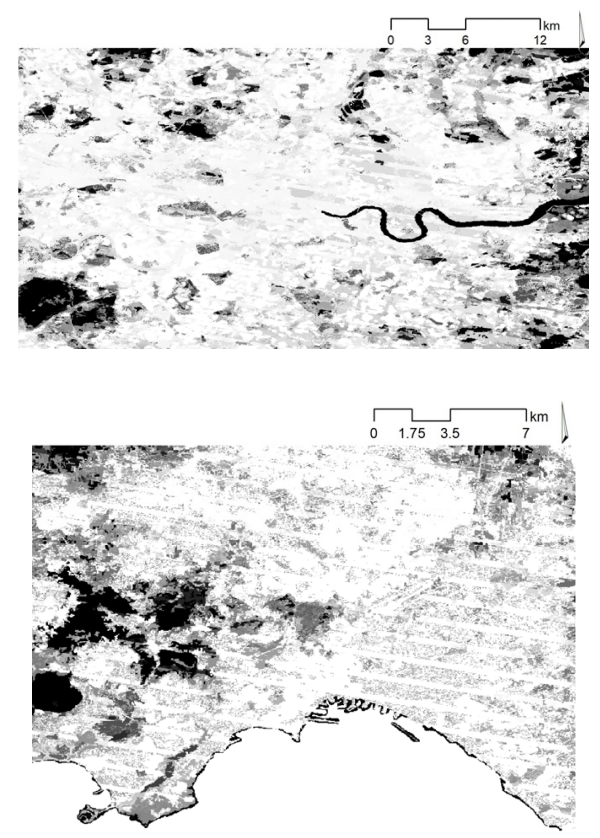

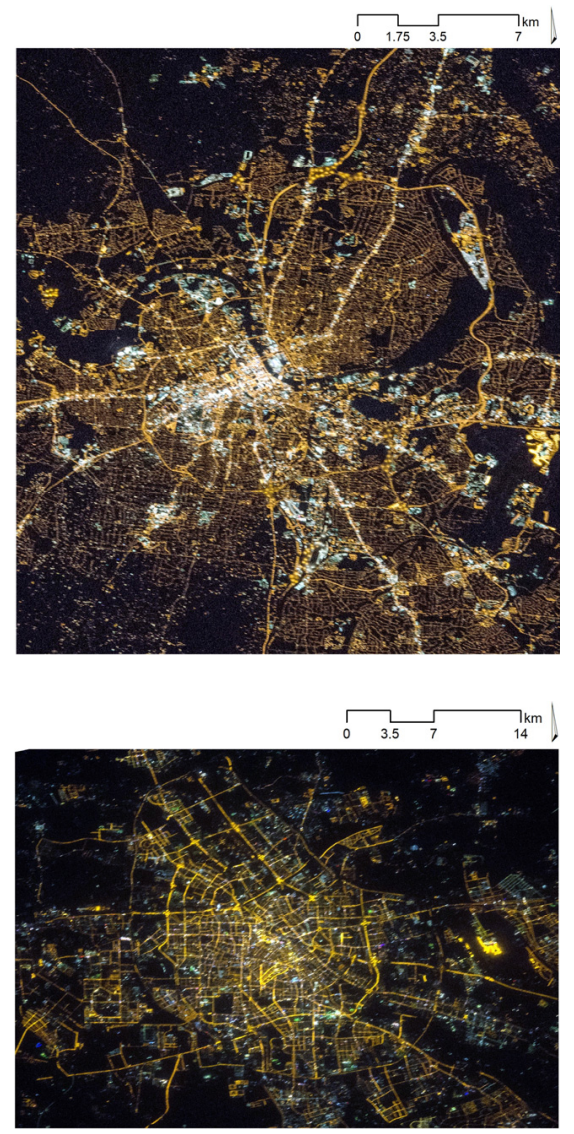

(a)

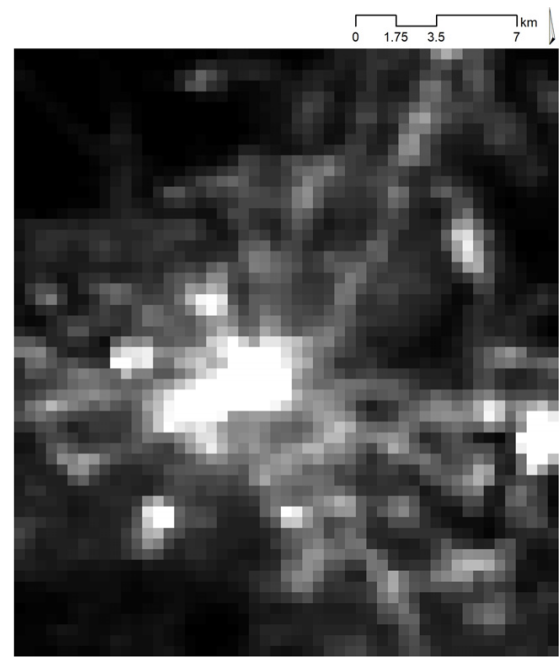

Nashville (ISS045-E-162944)

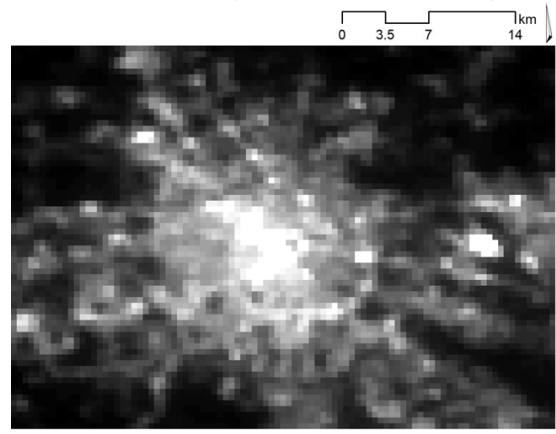

Tianjin (ISS047-E-12004)

(b)
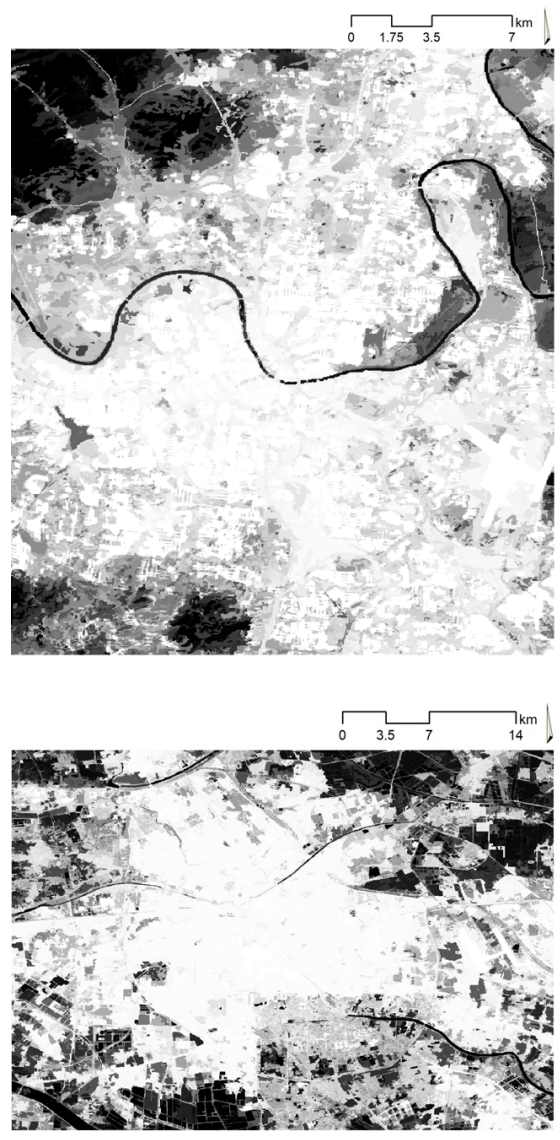

(c)

Figure 1. Raw input data for metropolitan areas under analysis: RGB NTL images (a), panchromatic NTL images (b), and HBASE images (c). Notes: RGB NTL images are $~ 10-\mathrm{m}$ resolution with the range of values of 0-255 digital numbers (dn) for each band; panchromatic NTL images are $~ 500-\mathrm{m}$ resolution with the values in the range of $1-740 \mathrm{nW} / \mathrm{cm}^{2} / \mathrm{sr}$; HBASE images are $\sim 30-\mathrm{m}$ resolution with the values in the $0-100 \%$ range. For the sake of comparability, RGB images, selected for analysis, were made by the same DSLR camera (Nikon D4), and their acquisition time is close to the VIIRS/DNB acquisition, that is, at about 01:30 a.m., local time.

\subsection{Data Processing}

To obtain inputs and outputs for neural networks, we used ArcGIS v.10.x software [55] to resample three types of the images (see Figure 1) to the resolution of the coarsest of them - that is, $\sim 500-\mathrm{m}$ resolution of panchromatic NTL data (Figure 2 reports processed images for the Haifa area, Israel). Thus, from the initial HBASE image (right panel in Figure 1), we form two 500-m resolution images, reporting average levels and standard deviations of initial middle-resolution HBASE data in each new coarse pixel (see Figure 2b,c). From each band of the initial high-resolution RGB image (left panel of Figure 1) we form a 500-m resolution image, reporting average levels or red, green, and blue band lights in each corresponding new coarse pixel (see correspondingly Figure $2 \mathrm{~d}-\mathrm{f}$ ).

Afterward, using MATLAB v.R2020x software [56], in order to obtain inputs for the convolutional neural networks, we have sliced all the resampled layers (Figure 2) using a kernel with the unit stride into small fragments of $K \times K$ size, $K \epsilon[3 ; 5 ; 7 ; 9 ; 11 ; 13 ; 15]$. Figure 3 reports an example of $7 \times 7$ fragments of the resampled layers for the Haifa region. At that, fragments of panchromatic NTL, average and standard deviation levels of HBASE (Figure $3 \mathrm{a}-\mathrm{c}$ ) formed three layers of input images for $\mathrm{CNN}$, while the values of central pixels from the red, green, or blue light fragments (Figure $3 \mathrm{~d}-\mathrm{f}$ ) consequently emerged as dependent variables. 


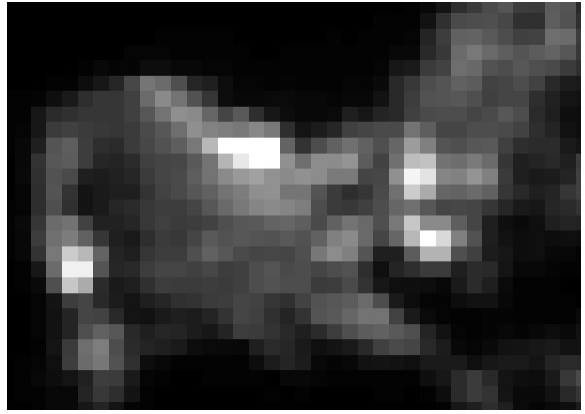

(a)

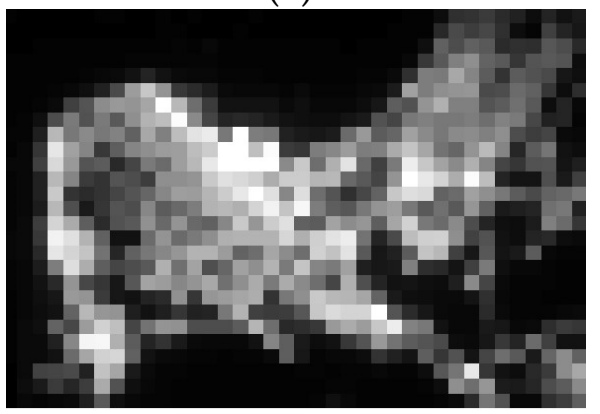

(d)

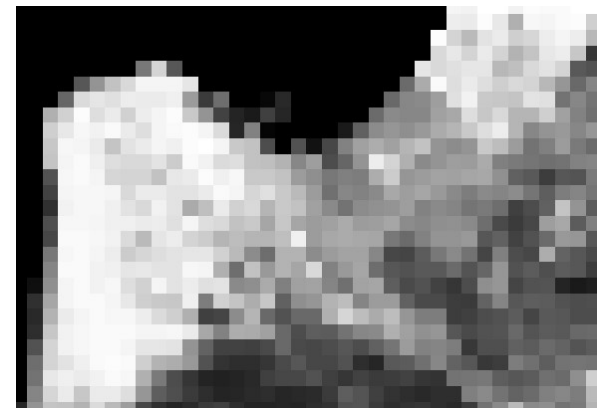

(b)

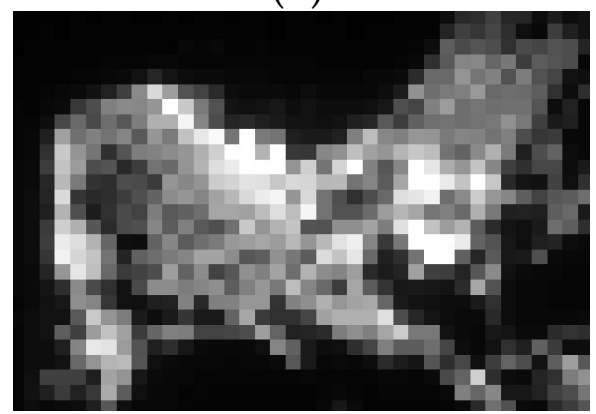

(e)

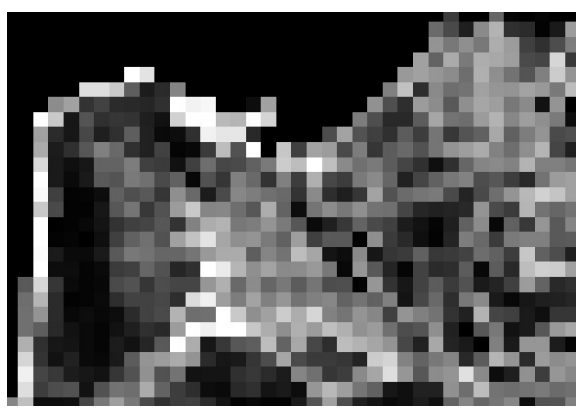

(c)

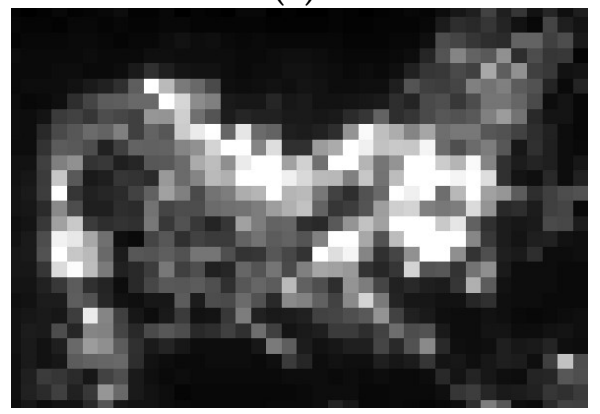

(f)

Figure 2. Resampled input data (to the resolution of $\sim 500-\mathrm{m}$ ) for the Haifa region (Israel). (a) Panchromatic NTL; (b) Average levels of HBASE; (c) Std. Dev. levels of HBASE; (d) Average red light levels; (e) Average green light levels; (f) Average blue light levels.

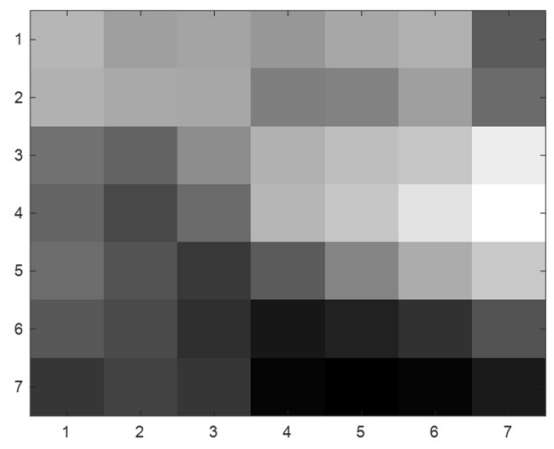

(a)

\section{Red Level = 145}

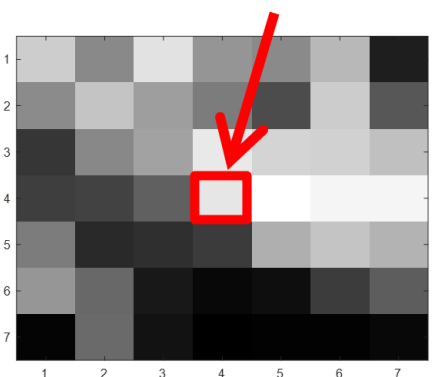

(d)

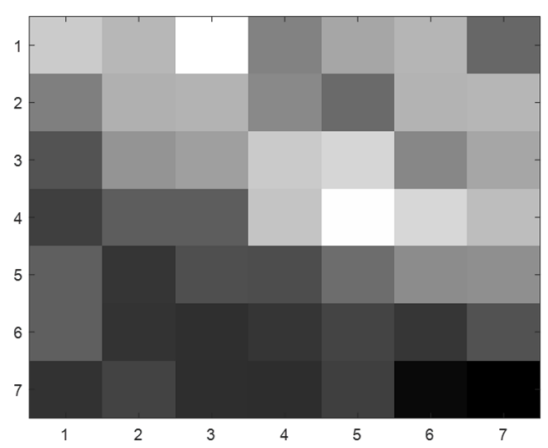

(b)
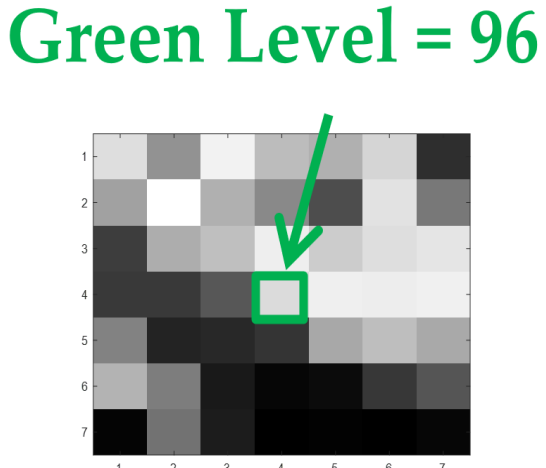

(e)

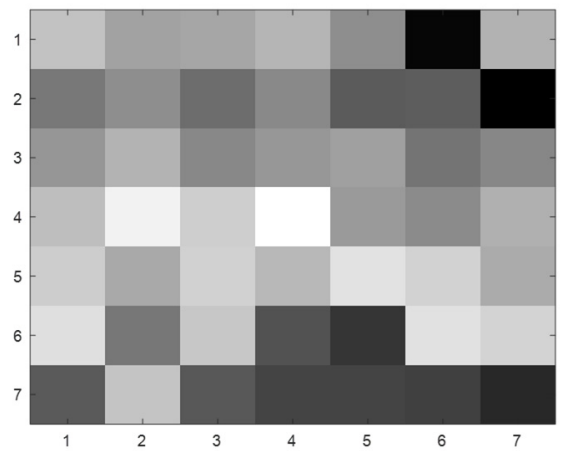

(c)
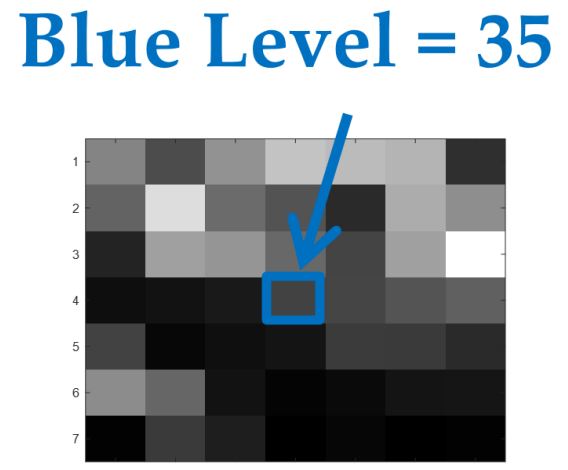

(f)

Figure 3. A unit of the CNN input data: Three layers simultaneously used as input images (a-c) and three color bands with the levels of central pixels (framed) used as alternative output labels (d-f). Note: The data stand for Haifa (Israel), the central pixel position is the 21st raw, 21st column. (a) Panchromatic NTL; (b) Average levels of HBASE; (c) Std. Dev. levels of HBASE; (d) Average red light levels; (e) Average green light levels; (f) Average blue light levels. 


\subsection{CNN Architecture}

Since we had small-scale input images (from only $3 \times 3$ to $15 \times 15$ pixels) of a relatively small number (see Table 1), training a heavy CNN seemed not appropriate [57]. Therefore, we performed trial experiments with several 'light-weight' CNN architectures and eventually selected the one depicted in Figure 4. The selected CNN architecture included the following consequent layers: (1) Input image layer, represented by the sets of input images of $K \times K$ size, $K \epsilon[3 ; 5 ; 7 ; 9 ; 11 ; 13 ; 15]$; (2) 3D convolutional layer (Conv3D) of $3 \times 3 \times 3$ size, followed by Batch Normalization (BN) and Rectified Linear Unit (ReLu) layers; (3) 2D convolutional layer (Conv2D) of $3 \times 3$ size, also followed by BN and ReLu layers; (4) one fully connected layer; and (5) regression layer, aimed to associate the output with continuous levels of either red, green, or blue light intensity.

For either red, green, or blue band light prediction, we trained CNN on the images of a certain metropolitan area and applied the obtained model to each of the rest cities. The analysis was performed in MATLAB v.R2020b software [58] using TrainNetwork function [59] with a default training options (i.e., under stochastic gradient descent with momentum $(\mathrm{SGDM})$ optimizer; minibatch size $=128$; initial learn rate $=0.001$; learn rate schedule $=$ piecewise; learn rate drop factor $=0.1$; learn rate drop period $=20$; shuffle $=$ every epoch).

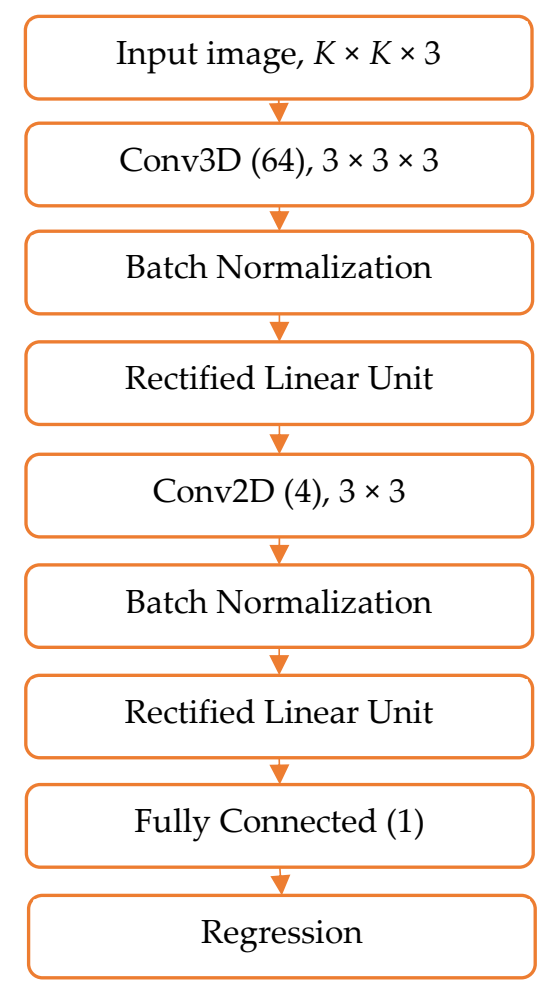

Figure 4. The architecture of the $\mathrm{CNN}$, used in the analysis. Note: Conv2D and followed by BN and ReLu layers were omitted in the case of $3 \times 3 \times 3$ input image size.

Table 1. The number of inputs for CNN depending on input image size.

\begin{tabular}{cccccccc}
\hline \multirow{2}{*}{ Region } & \multicolumn{7}{c}{ Input Image Size } \\
\cline { 2 - 8 } & $\mathbf{3} \times \mathbf{3}$ & $\mathbf{5 \times 5}$ & $\mathbf{7} \times \mathbf{7}$ & $\mathbf{9} \times \mathbf{9}$ & $\mathbf{1 1} \times \mathbf{1 1}$ & $\mathbf{1 3} \times \mathbf{1 3}$ & $\mathbf{1 5} \times \mathbf{1 5}$ \\
\hline Atlanta & 5609 & 5313 & 5025 & 4745 & 4473 & 4209 & 3953 \\
\hline Beijing & 5400 & 5088 & 4784 & 4488 & 4200 & 3920 & 3648 \\
\hline Haifa & 900 & 782 & 672 & 570 & 476 & 390 & 312 \\
\hline Khabarovsk & 3550 & 3312 & 3082 & 2860 & 2646 & 2440 & 2242 \\
\hline
\end{tabular}


Table 1. Cont.

\begin{tabular}{cccccccc}
\hline \multirow{2}{*}{ Region } & \multicolumn{7}{c}{ Input Image Size } \\
\cline { 2 - 8 } & $\mathbf{3} \times \mathbf{3}$ & $\mathbf{5} \times \mathbf{5}$ & $\mathbf{7} \times \mathbf{7}$ & $\mathbf{9} \times \mathbf{9}$ & $\mathbf{1 1} \times \mathbf{1 1}$ & $\mathbf{1 3} \times \mathbf{1 3}$ & $\mathbf{1 5} \times \mathbf{1 5}$ \\
\hline London & 4850 & 4560 & 4278 & 4004 & 3738 & 3480 & 3230 \\
\hline Naples & 1872 & 1700 & 1536 & 1380 & 1232 & 1092 & 960 \\
\hline Nashville & 2695 & 2491 & 2295 & 2107 & 1927 & 1755 & 1591 \\
\hline Tianjing & 6045 & 5733 & 5429 & 5133 & 4845 & 4565 & 4293 \\
\hline
\end{tabular}

\subsection{Assessing the Quality of the Models}

The performance of the models for training and testing sets of images was assessed via two indicators: (i) Pearson's correlation and (ii) weighted mean squared error (WMSE) between actual and model-estimated red, green, or blue band light intensity. The first indicator is aimed to assess the model's ability to produce RGB estimates, which in their relative tendency correspond well with the observed RGB levels, while the second one, calculated as mean squared difference between the model-estimated and actually observed RGB levels, divided by the actually observed value, helps to assess differences between the estimated and actual RGB levels on an absolute scale. Statistical analysis was performed using JASP v.0.14.x software [60].

\section{Results}

\subsection{Neighborhood Effect: Models' Performance upon Training and Testing Datasets}

Visual inspection witnesses fairly sufficient similarity between the original RGB levels and those restored using the proposed CNN models, although the restored images look slightly more blurred. Figures 5 and 6 report such pairs for R, G, and B bands for two cities, representing small (Haifa, Israel) and large (London, UK) metropolitan areas.

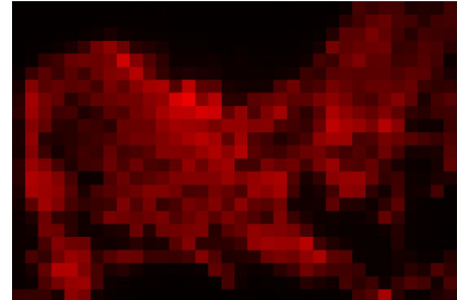

(a)

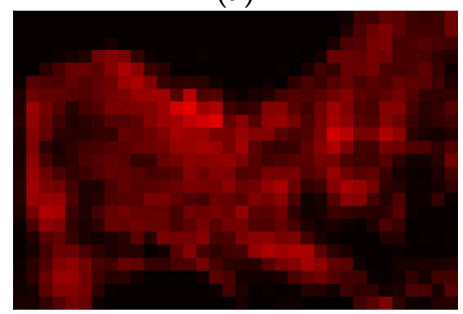

(d)

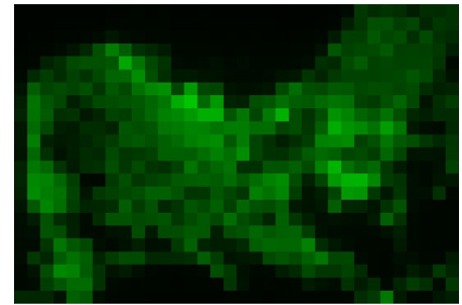

(b)

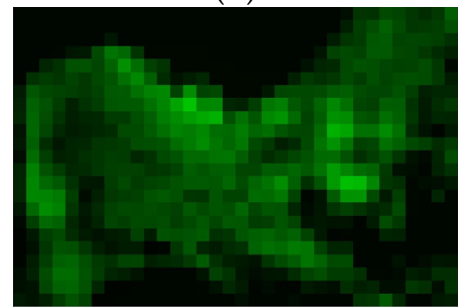

(e)

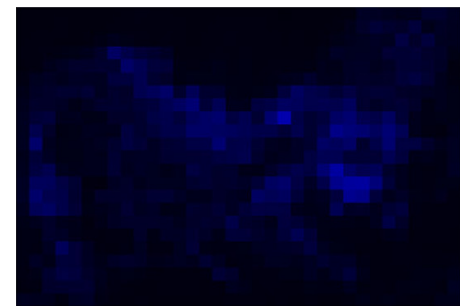

(c)

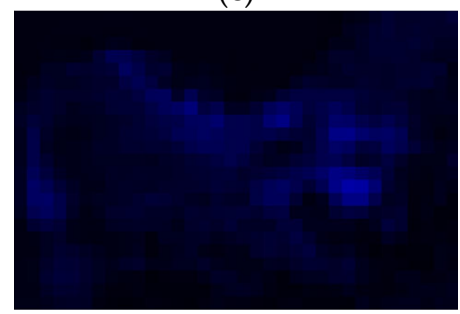

(f)

Figure 5. Original vs. CNN-estimated $(k=5)$ RGB levels in Haifa area: (a) original red; (b) original green; (c) original blue; (d) estimated red; (e) estimated green; (f) estimated blue. Note: The images are of panchromatic NTL spatial resolution.

In Figure 7, we report the effect of the input image size upon two performance indicators-Pearson's correlation and WMSE for each color band, separately for (eight) training and (56, i.e., seven for each of the eight training) testing sets. As one can see from the figures, both indicators' levels, averaged across training sets (Figure 7a,b), changed non-monotonically, with the extremum at $5 \times 5$ image size, for any color band prediction. To check whether the input size $5 \times 5$ is indeed the extremum, we conducted a one-tailed paired samples $t$-test for the statistical significance of the difference of means-against both 
smaller $(3 \times 3)$ and larger $(7 \times 7)$ input sizes. For all channels, mean levels of Pearson's correlation for $5 \times 5$ input image size were significantly higher than the ones of either $3 \times 3$ and $7 \times 7$ input image size $(|t|>2.335 ; p<0.026$ for the red channel; $|t|>2.404 ; p<0.024$ for the green channel; and $|t|>2.531 ; p<0.020$ for the blue channel). In the meantime, the pattern in terms of WMSE levels was something less pronounced: $5 \times 5$ input image size was always significantly better than $3 \times 3$ image $(|t|=3.227 ; p=0.007$ for red channel; $|t|=2.812 ; p=0.013$ for green channel; and $|t|=3.126 ; p=0.008$ for blue channel), but not always better than $7 \times 7$ counterpart $(|t|=1.228 ; p=0.130$ for red channel; $|t|=1.884 ;$ $p=0.051$ for green channel; and $|t|=0.962 ; p=0.184$ for blue channel).

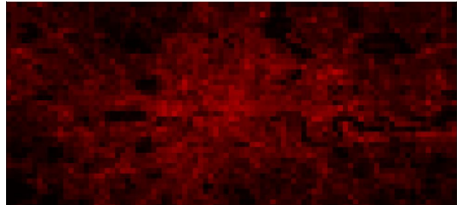

(a)

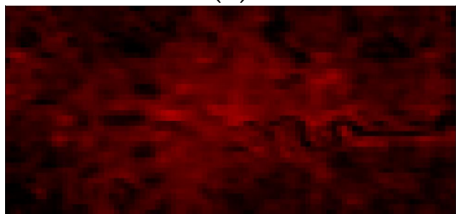

(d)

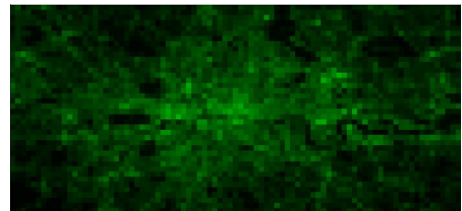

(b)

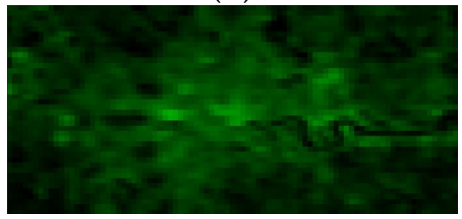

(e)

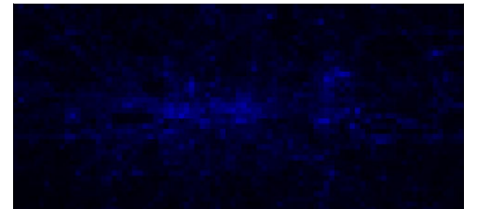

(c)

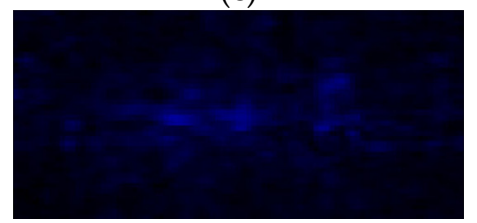

(f)

Figure 6. Original vs. CNN-estimated ( $k=13)$ RGB levels in London area: (a) original red; (b) original green; (c) original blue; (d) estimated red; (e) estimated green; (f) estimated blue. Note: The images are of panchromatic NTL spatial resolution.

We should however notice that-if to analyze the models' performance indicators for each training dataset separately-optimal input image size is relatively small for smaller, either in terms of area or population, metropolitan areas $(5 \times 5$ image size is optimal for Haifa (with $\sim 0.3 \mathrm{mln}$ people population), Khabarovsk ( $\sim .6 \mathrm{mln}$ people), Nashville ( $\sim 0.7 \mathrm{mln}$ people), and Naples ( $3 \mathrm{mln}$ people $)$ ), and larger for bigger metropolitan areas (input images of generally greater than $7 \times 7$ size are optimal for Atlanta (with $\sim 5.5 \mathrm{mln}$ population), London ( $\sim 9 \mathrm{mln}$ people), Tianjin ( $\sim 15 \mathrm{mln}$ people), and Beijing ( $21.5 \mathrm{mln}$ people)). Interestingly, for bigger areas, there emerges differentiation in optimal input image size for different color band intensities prediction. Generally, for short-wavelength blue lights, the optimal input image size was smaller than for long-wavelength red lights (for instance, $5 \times 5$ and $7 \times 7$ optimal image size for blue lights and $11 \times 11$ and $13 \times 13$ for red lights correspondingly in Beijing and Atlanta). In Figure 8 we report models performance indicators for two selected training datasets, representing typical relatively small (Nashville-see Figure 8a,b) and big (Atlanta-see Figure 8c,d) metropolitan areas.

We should note that for testing datasets, the tendency in the models' performance indicators is less pronounced (Figure $7 c, d$ ), perhaps due to averaging across a greater amount of heterogeneous datasets. Visually, the models generally demonstrate the best performance upon $3 \times 3$ input images. Although, the difference with $5 \times 5$ input image size is not always statistically significant. Due to violation of normality, we replaced the paired sample $t$-test for the statistical significance of the difference of means by non-parametric Wilcoxon signed-rank test for the difference of medians. For red and green channels, we found a significant difference in terms of Pearson's correlation $(p<0.001)$ but not WMSE $(p>0.204)$; In contrast, for the blue channel, the difference appeared significant in terms of WMSE $(p<0.001)$ but not Pearson's correlation $(p>0.801)$. In the meantime, a more detailed examination shows that models built for smaller metropolitan areas (Haifa, Khabarovsk, Naples, and Nashville) generally fit better other relatively small areas regardless of input image size; While models built for relatively large metropolitan areas (Atlanta, Beijing, London, and Tianjin) fit better other large areas given large enough input image size (not shown). 


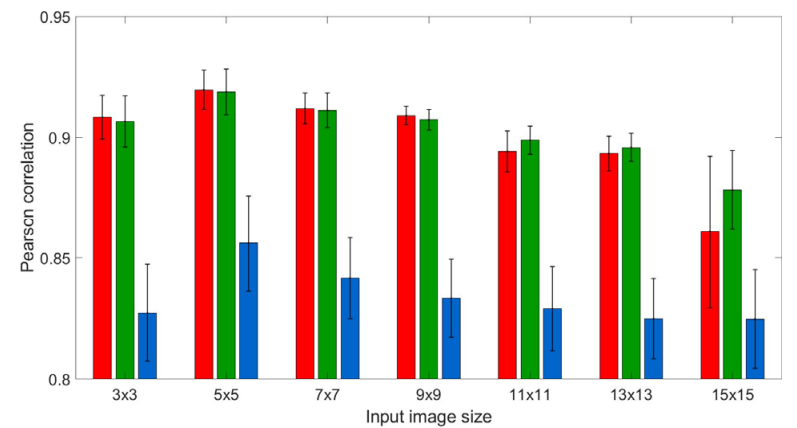

(a)

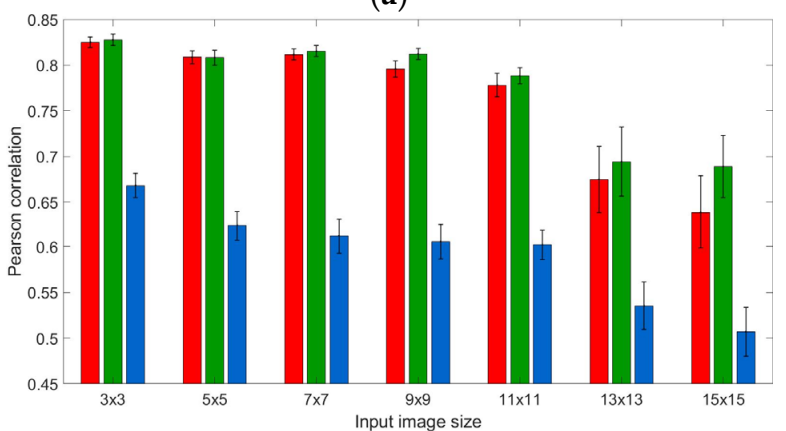

(c)

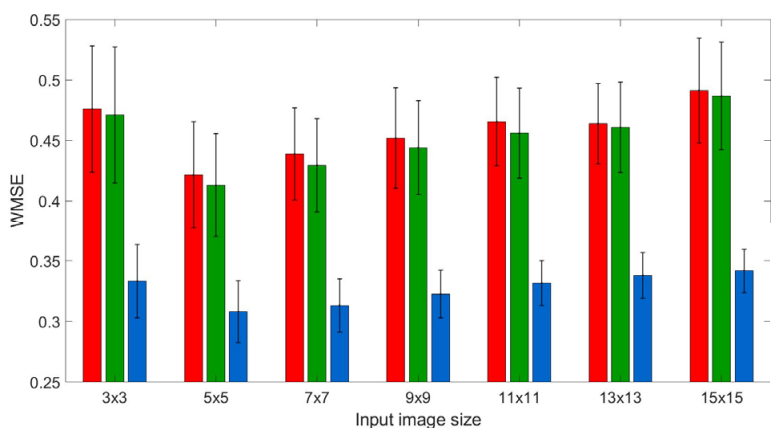

(b)

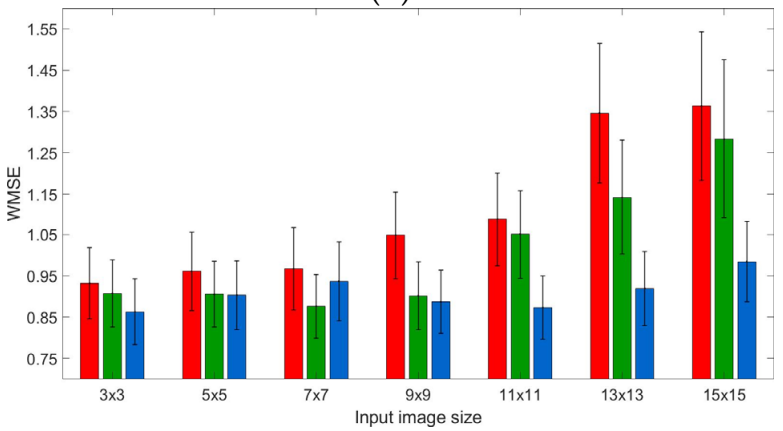

(d)

Figure 7. The effects of input image size on CNN performance for the training (top row) and testing (bottom row) datasets, in terms of averaged Pearson's correlation coefficients $(\mathbf{a}, \mathbf{c})$, and WMSE $(\mathbf{b}, \mathbf{d})$. Notes: In case of Pearson's correlation $(\mathbf{a}, \mathbf{c})$, greater means better; In case of WMSE $(\mathbf{b}, \mathbf{d})$, lower means better. Error lines stand for the standard error of the mean.

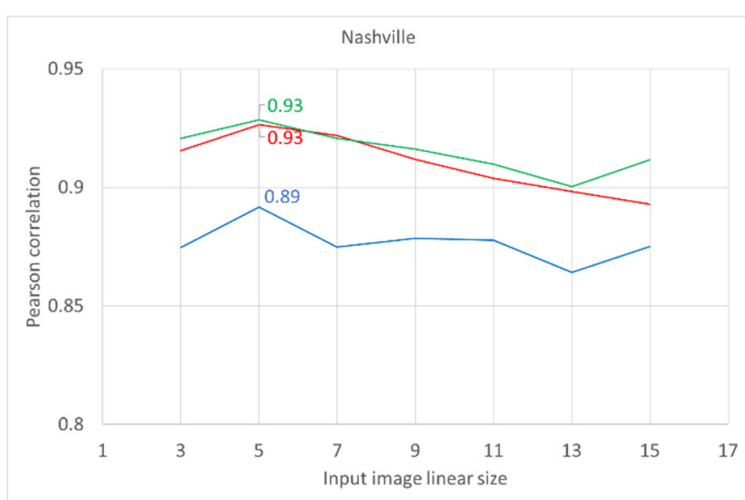

(a)

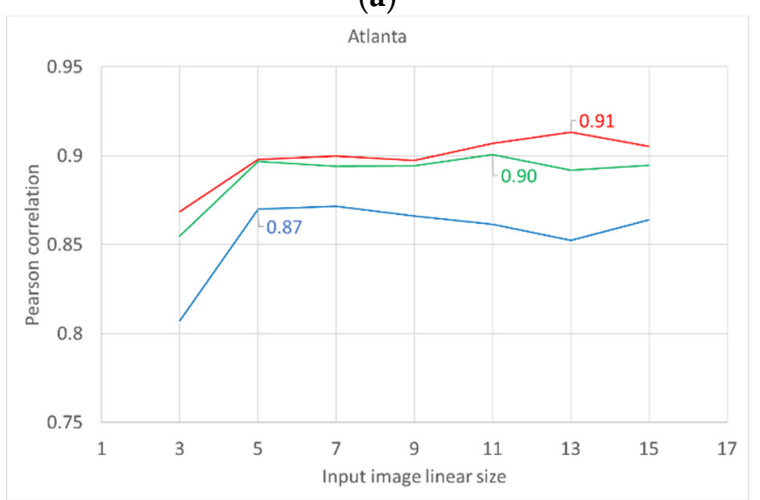

(c)

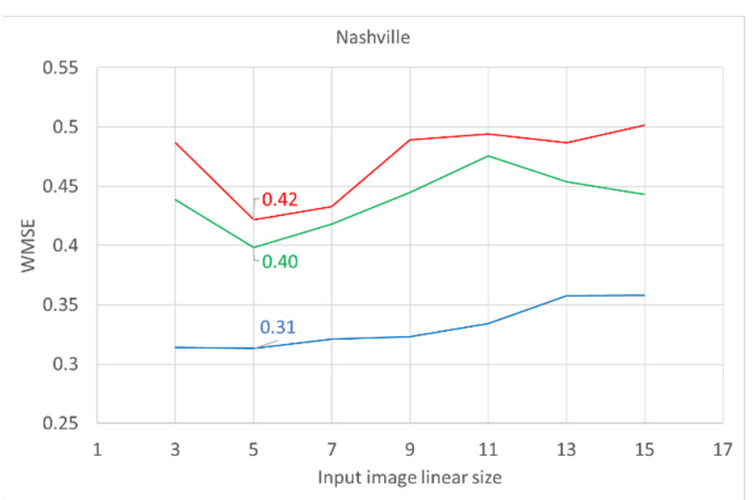

(b)

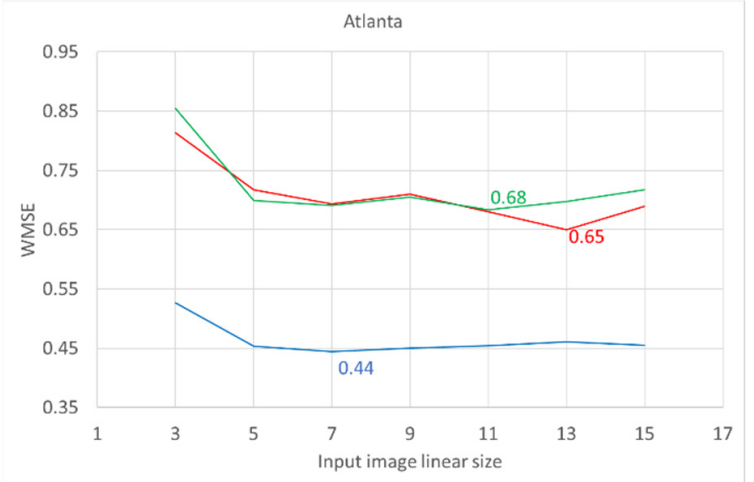

(d)

Figure 8. The effects of input image size on the performance of CNN models built for selected training datasets, in terms of Pearson's correlation coefficients (left panel), and WMSE (right panel). Notes: In the case of Pearson's correlation (a,c), greater means better; In the case of WMSE $(\mathbf{b}, \mathbf{d})$, lower means better; the best values are labeled. 


\subsection{CNN Models Comparison with Other Machine Learning Techniques}

We should also note that the best-performing CNN models (with $5 \times 5$ input images) demonstrate comparable performance with previously explored techniques [27] in terms of Pearson's correlations both for training and testing datasets (Figure 9a,c,e), but, compared to other techniques, perform much more stable in terms of WMSE upon training and testing sets-the ratios are within 2.20-2.95 diapason for $\mathrm{CNN}$ vs. 3.76-22.78 for other techniques (Figure 9f).

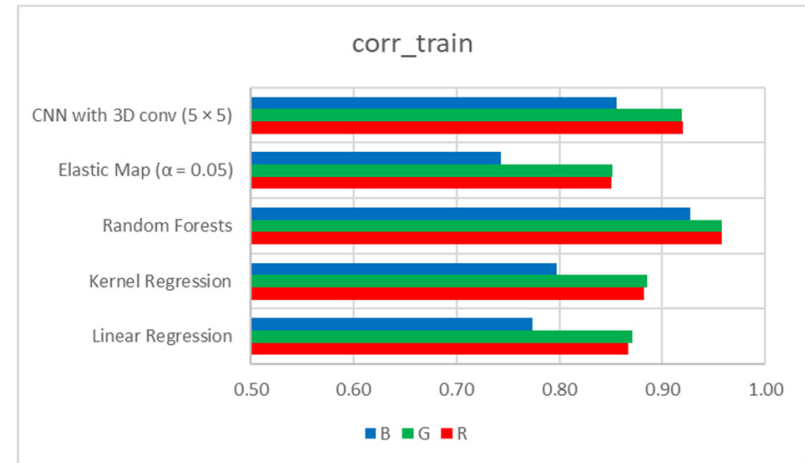

(a)

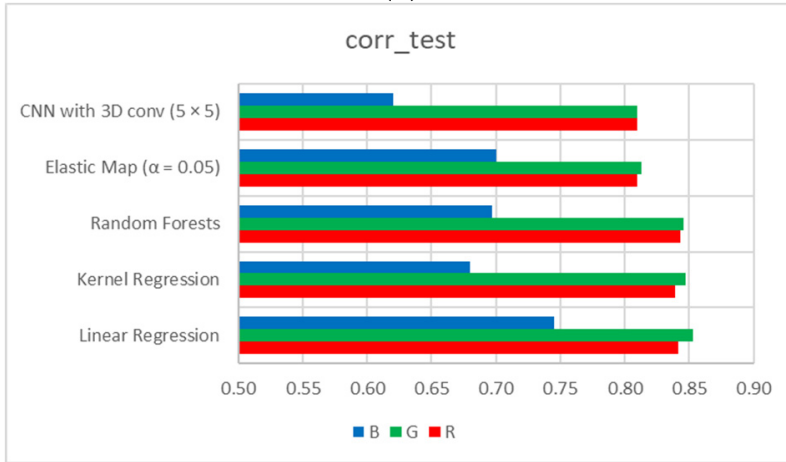

(c)

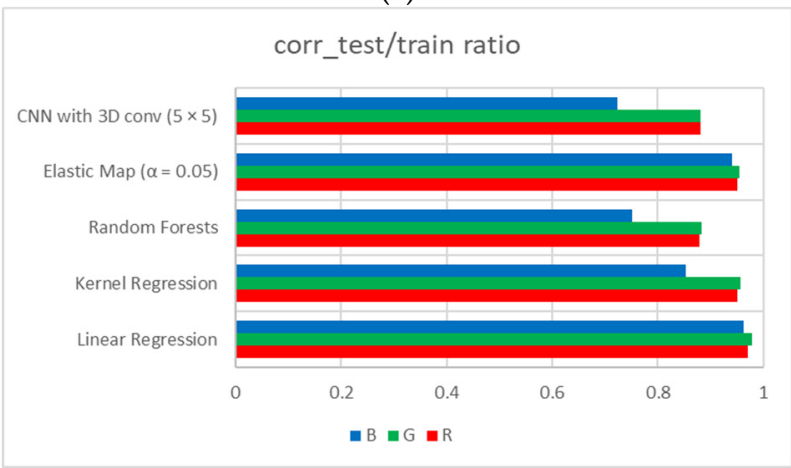

(e)

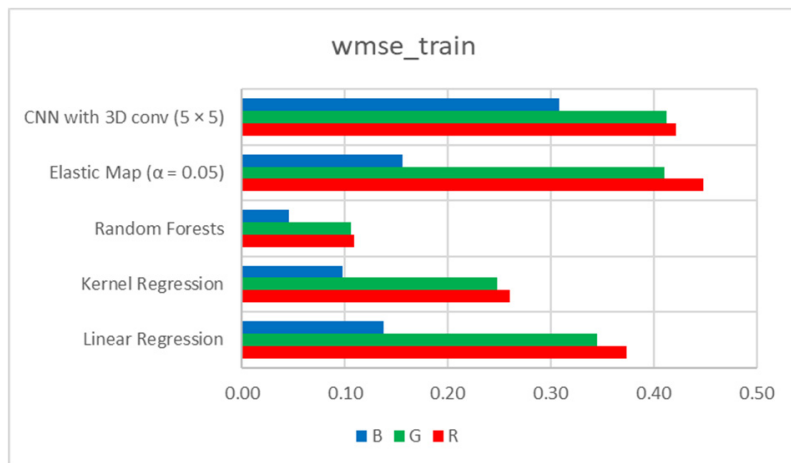

(b)

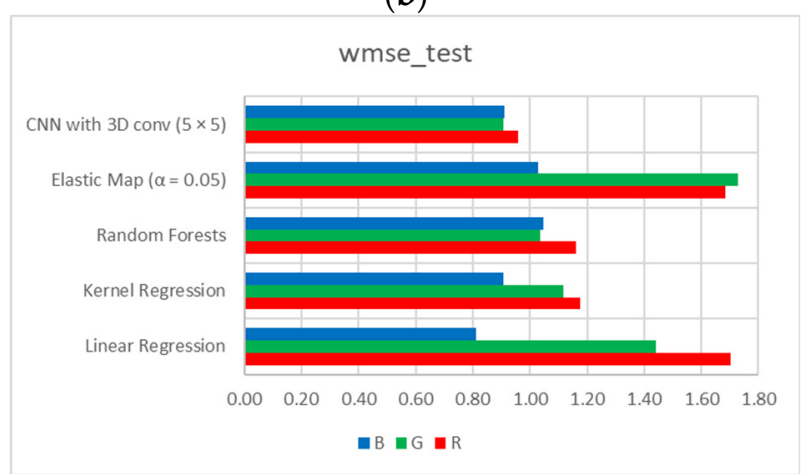

(d)

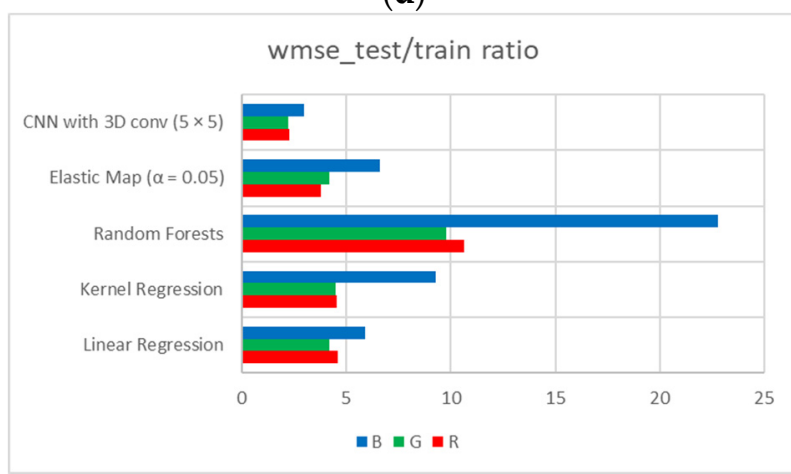

$(\mathbf{f})$

Figure 9. Mutual comparison of linear and kernel regressions, random forest, elastic map, and best-performing CNN models for the training (top row) and testing (bottom row) datasets, in terms of averaged Pearson's correlation coefficients $(\mathbf{a}, \mathbf{c})$, and WMSE $(\mathbf{b}, \mathbf{d})$. Note: In case of Pearson's correlation $(\mathbf{a}, \mathbf{c})$, greater means better; In case of WMSE (b,d), lower means better.

\section{Discussion and Conclusions}

The present study aimed at restoring RGB intensities, reported by the ISS [25], from VIIRS-DNB-provided panchromatic night-time lights imagery [17] and the levels of the built-up area [54], used as a proxy for land-use types. We used corresponding datasets for eight metropolitan areas: Atlanta (US), Beijing (China), Haifa (Israel), Khabarovsk (Russia), 
London (UK), Naples (Italy), Nashville (US), and Tianjin (China), as case studies. To restore the RGB level of the pixel under analysis, we used panchromatic NTL and built-up area levels from varying neighborhoods, starting from the first-order neighboring pixels and up to the neighbors of the seventh level. Thus, for each of the eight metropolitan areas, we sliced input layers for small-scale squared fragments using a kernel with unit stride, and consequently-for each input image size dataset-run CNN models, using either red, green, or blue light level of the central pixel from the corresponding fragment as a dependent variable. Each model, built for a certain metropolitan area dataset, was validated over the rest seven cities.

As our analysis revealed, for relatively small metropolitan areas, either in terms of area or population (such as Haifa, Khabarovsk, Naples, and Nashville), the best-performing models for any color light band prediction were built for $5 \times 5$ input image size, while for larger ones (such as Atlanta, Beijing, London, and Tianjin) the optimal input image size was at least $7 \times 7$ pixels. A speculative explanation is on the average larger physical extent of residential quarters, commercial and entertainment centers, and industrial facilities in larger cities. Yet, this assumption should be explicitly tested.

Another important finding of the present study is that for bigger metropolitan areas emerges differentiation in optimal input image size for different color band lights prediction. Generally, for long-wavelength red lights, the optimal input image size is larger than for short-wavelength blue lights (compare $11 \times 11$ and $13 \times 13$ pixels optimal image size for red lights and $5 \times 5$ and $7 \times 7$ pixels for blue lights correspondingly in Beijing and Atlanta). There exist several indications that red and green lights are more associated with residential areas, while industrial and commercial facilities are often lit by blue lights [19,61]. Since residential areas are usually more extensive, while industrial and commercial facilities are localized, red and green lights are expected to be predicted better by larger neighborhoods. In the meantime, this tendency was not confirmed by the case of London (with $9 \times 9$ and $13 \times 13$ optimal input image size for red and blue lights, correspondingly) and Tianjin (with the same $7 \times 7$ optimal input image size for all colored lights prediction) and thus requires further investigation. Other directions of future investigation might include experiments with a larger amount of metropolitan areas and alternative $\mathrm{CNN}$ architectures.

To compare different machine learning techniques, none of them demonstrated an absolute advantage over its counterparts: Instead, the advantage depended on the chosen performance indicator. Compared to our previous study, where we examined first-order neighborhood effect only and used several machine learning techniques, such as linear regression, non-linear kernel regression, random forest, and elastic map models [27], CNN models performed something worse in terms of Pearson's correlation upon testing sets. In this sense, it seems perspective to seek for $\mathrm{CNN}$ architectures less sensitive to data heterogeneity. At the same time, compared to other machine learning techniques, CNN models showed better performance in terms of WMSE for testing datasets for red ( 0.91 for CNN models vs. 1.04-1.73 for other machine learning techniques) and green ( 0.96 for $\mathrm{CNN}$ models vs. 1.16-1.70 for other machine learning techniques) bands prediction. We explain such an improvement by accounting for the varying neighborhood effect, which is more important for relatively vaster residential area-associated bands $[19,61]$.

Author Contributions: Conceptualization, A.N.G.; methodology, N.R., E.M.M. and A.N.G.; software, N.R. and E.M.M.; validation, N.R.; formal analysis, N.R.; investigation, N.R., E.M.M. and A.N.G.; resources, N.R.; data curation, N.R.; writing—original draft preparation, N.R.; writing-review and editing, E.M.M. and A.N.G.; visualization, N.R.; supervision, A.N.G.; project administration, N.R.; funding acquisition, A.N.G. All authors have read and agreed to the published version of the manuscript.

Funding: This research was supported by the Council for Higher Education of Israel (postdoctoral scholarship of N.R.) and by the Ministry of Science and Higher Education of the Russian Federation (Project No. 075-15-2021-634).

Institutional Review Board Statement: Not applicable. 


\section{Informed Consent Statement: Not applicable.}

Data Availability Statement: The data and the code presented in this study are openly available from https:/ / github.com/Mirkes/Sensor-paper-for-CNN-based-image-colouring. (accessed on 2 November 2021).

Acknowledgments: N.R. is deeply thankful to Sviatoslav Rybnikov for the inspiring discussion and valuable comments. The authors are grateful to three anonymous reviewers for their useful comments and suggestions.

Conflicts of Interest: The authors declare no conflict of interest. The funders had no role in the design of the study; in the collection, analyses, or interpretation of data; in the writing of the manuscript, or in the decision to publish the results.

\section{References}

1. Elvidge, C.D.; Baugh, K.E.; Kihn, E.A.; Kroehl, H.W.; Davis, E.R.; Davis, C.W. Relation between satellite observed visible-near infrared emissions, population, economic activity and electric power consumption. Int. J. Remote Sens. 1997, 18, 1373-1379. [CrossRef]

2. Sutton, P.; Roberts, D.; Elvidge, C.; Baugh, K. Census from Heaven: An estimate of the global human population using night-time satellite imagery. Int. J. Remote Sens. 2001, 22, 3061-3076. [CrossRef]

3. Doll, C.N.H.; Muller, J.P.; Morley, J.G. Mapping regional economic activity from night-time light satellite imagery. Ecol. Econ. 2006, 57, 75-92. [CrossRef]

4. Ebener, S.; Murray, C.; Tandon, A.; Elvidge, C.C. From wealth to health: Modelling the distribution of income per capita at the sub-national level using night-time light imagery. Int. J. Health Geogr. 2005, 4, 5. [CrossRef]

5. Ghosh, T.; Powell, R.L.; Elvidge, C.D.; Baugh, K.E.; Sutton, P.C.; Anderson, S. Shedding light on the global distribution of economic activity. Open Geogr. J. 2010, 3, 147-160.

6. Mellander, C.; Lobo, J.; Stolarick, K.; Matheson, Z. Night-time light data: A good proxy measure for economic activity? PLoS ONE 2015, 10, e0139779.

7. Hopkins, G.R.; Gaston, K.J.; Visser, M.E.; Elgar, M.A.; Jones, T.M. Artificial light at night as a driver of evolution across urban-rural landscapes. Front. Ecol. Environ. 2018, 16, 472-479. [CrossRef]

8. Tselios, V.; Stathakis, D. Exploring regional and urban clusters and patterns in Europe using satellite observed lighting. Environ. Plan. B Urban Anal. City Sci. 2018, 47, 553-568. [CrossRef]

9. Kloog, I.; Haim, A.; Stevens, R.G.; Portnov, B.A. Global co-distribution of light at night (LAN) and cancers of prostate, colon, and lung in men. Chronobiol. Int. 2009, 26, 108-125. [CrossRef] [PubMed]

10. Kloog, I.; Stevens, R.G.; Haim, A.; Portnov, B.A. Nighttime light level co-distributes with breast cancer incidence worldwide. Cancer Causes Control 2010, 21, 2059-2068. [CrossRef]

11. Rybnikova, N.A.; Haim, A.; Portnov, B.A. Does artificial light-at-night exposure contribute to the worldwide obesity pandemic? Int. J. Obes. 2016, 40, 815-823. [CrossRef]

12. Bennie, J.; Duffy, J.; Davies, T.; Correa-Cano, M.; Gaston, K. Global Trends in Exposure to Light Pollution in Natural Terrestrial Ecosystems. Remote Sens. 2015, 7, 2715-2730. [CrossRef]

13. Hu, Z.; Hu, H.; Huang, Y. Association between nighttime artificial light pollution and sea turtle nest density along Florida coast: A geospatial study using VIIRS remote sensing data. Environ. Pollut. 2018, 239, 30-42. [CrossRef]

14. Cinzano, P.; Falchi, F.; Elvidge, C.D.; Baugh, K.E. The artificial night sky brightness mapped from DMSP satellite Operational Linescan System measurements. Mon. Not. R. Astron. Soc. 2000, 318, 641-657. [CrossRef]

15. Falchi, F.; Cinzano, P.; Duriscoe, D.; Kyba, C.C.M.; Elvidge, C.D.; Baugh, K.; Portnov, B.A.; Rybnikova, N.A.; Furgoni, R. The new world atlas of artificial night sky brightness. Sci. Adv. 2016, 2, e1600377. [CrossRef] [PubMed]

16. Falchi, F.; Furgoni, R.; Gallaway, T.A.A.; Rybnikova, N.A.A.; Portnov, B.A.A.; Baugh, K.; Cinzano, P.; Elvidge, C.D.D. Light pollution in USA and Europe: The good, the bad and the ugly. J. Environ. Manag. 2019, 248, 109227. [CrossRef]

17. Earth Observation Goup. Available online: https://eogdata.mines.edu/products/vnl/ (accessed on 8 August 2021).

18. Elvidge, C.D.; Baugh, K.E.; Zhizhin, M.; Hsu, F.-C. Why VIIRS data are superior to DMSP for mapping nighttime lights. Proc. Asia-Pac. Adv. Netw. 2013, 35, 62. [CrossRef]

19. Rybnikova, N.A.; Portnov, B.A. Remote identification of research and educational activities using spectral properties of nighttime light. ISPRS J. Photogramm. Remote Sens. 2017, 128, 212-222. [CrossRef]

20. Veitch, J.; Newsham, G.; Boyce, P.; Jones, C. Lighting appraisal, well-being and performance in open-plan offices: A linked mechanisms approach. Light. Res. Technol. 2008, 40, 133-151. [CrossRef]

21. Guk, E.; Levin, N. Analyzing spatial variability in night-time lights using a high spatial resolution color Jilin-1 image-Jerusalem as a case study. ISPRS J. Photogramm. Remote Sens. 2020, 163, 121-136. [CrossRef]

22. Cajochen, C.; Münch, M.; Kobialka, S.; Kräuchi, K.; Steiner, R.; Oelhafen, P.; Orgül, S.; Wirz-Justice, A. High Sensitivity of Human Melatonin, Alertness, Thermoregulation, and Heart Rate to Short Wavelength Light. J. Clin. Endocrinol. Metab. 2005, 90, 1311-1316. [CrossRef] [PubMed] 
23. Haim, A.; Portnov, B.A. Light Pollution As a New Risk Factor for Human Breast and Prostate Cancers; Springer: Dordrecht, The Netherlands, 2013; ISBN 9789400762206.

24. McFadden, E.; Jones, M.E.; Schoemaker, M.J.; Ashworth, A.; Swerdlow, A.J. The relationship between obesity and exposure to light at night: Cross-sectional analyses of over 100,000 women in the Breakthrough Generations Study. Am. J. Epidemiol. 2014, 180, 245-250. [CrossRef]

25. Search Photos. Available online: https://eol.jsc.nasa.gov/SearchPhotos/ (accessed on 7 April 2020).

26. Jilin-1 Optical-A (Jilin-1 Guangxe-A)_Gunter's Space Page. Available online: https://space.skyrocket.de/doc_sdat/jilin-1optical-a.htm (accessed on 7 April 2020).

27. Rybnikova, N.; Portnov, B.A.; Mirkes, E.M.; Zinovyev, A.; Brook, A.; Gorban, A.N. Coloring Panchromatic Nighttime Satellite Images: Comparing the Performance of Several Machine Learning Methods. IEEE Trans. Geosci. Remote Sens. 2021. [CrossRef]

28. Wang, Z.; Chen, J.; Hoi, S.C.H. Deep Learning for Image Super-Resolution: A Survey. IEEE Trans. Pattern Anal. Mach. Intell. 2020, 43, 3365-3387. [CrossRef]

29. Milanfar, P. (Ed.) Super-Resolution Imaging; CRC Press: Boca Raton, FL, USA, 2017.

30. Tian, J.; Ma, K.-K. A survey on super-resolution imaging. Signal Image Video Process. 2011, 5, 329-342. [CrossRef]

31. Nasrollahi, K.; Moeslund, T.B. Super-resolution: A comprehensive survey. Mach. Vis. Appl. 2014, 25, 1423-1468. [CrossRef]

32. Park, S.C.; Park, M.K.; Kang, M.G. Super-resolution image reconstruction: A technical overview. IEEE Signal Process. Mag. 2003, 20, 21-36. [CrossRef]

33. Dong, C.; Loy, C.C.; He, K.; Tang, X. Image Super-Resolution Using Deep Convolutional Networks. IEEE Trans. Pattern Anal. Mach. Intell. 2016, 38, 295-307. [CrossRef] [PubMed]

34. Cheong, J.Y.; Park, I.K. Deep CNN-Based Super-Resolution Using External and Internal Examples. IEEE Signal Process. Lett. 2017, 24, 1252-1256. [CrossRef]

35. Xiao, A.; Wang, Z.; Wang, L.; Ren, Y. Super-Resolution for "Jilin-1" Satellite Video Imagery via a Convolutional Network. Sensors 2018, 18, 1194. [CrossRef]

36. Zhang, X.; Li, C.; Meng, Q.; Liu, S.; Zhang, Y.; Wang, J. Infrared Image Super Resolution by Combining Compressive Sensing and Deep Learning. Sensors 2018, 18, 2587. [CrossRef] [PubMed]

37. Wang, X.; Wu, Y.; Ming, Y.; Lv, H. Remote Sensing Imagery Super Resolution Based on Adaptive Multi-Scale Feature Fusion Network. Sensors 2020, 20, 1142. [CrossRef] [PubMed]

38. Tian, C.; Xu, Y.; Zuo, W.; Zhang, B.; Fei, L.; Lin, C.W. Coarse-to-Fine CNN for Image Super-Resolution. IEEE Trans. Multimed. 2021, 23, 1489-1502. [CrossRef]

39. Arun, P.V.; Buddhiraju, K.M.; Porwal, A.; Chanussot, J. CNN based spectral super-resolution of remote sensing images. Signal Process. 2020, 169, 107394. [CrossRef]

40. Can, Y.B.; Timofte, R. An efficient CNN for spectral reconstruction from RGB images. arXiv 2018, arXiv:1804.04647.

41. Galliani, S.; Lanaras, C.; Marmanis, D.; Baltsavias, E.; Schindler, K.; Sensing, R.; Zurich, E. Learned Spectral Super-Resolution. arXiv 2017, arXiv:1703.09470.

42. Aeschbacher, J.; Wu, J.; Timofte CVL, R.; Zurich, E. In Defense of Shallow Learned Spectral Reconstruction from RGB Images. In Proceedings of the IEEE International Conference on Computer Vision Workshops, Venice, Italy, 22-29 October 2017.

43. Arad, B.; Ben-Shahar, O. Sparse Recovery of Hyperspectral Signal from Natural RGB Images. In Proceedings of the 14th European Conference, Amsterdam, The Netherlands, 11-14 October 2016. [CrossRef]

44. Timofte, R.; De Smet, V.; Van Gool, L. Anchored Neighborhood Regression for Fast Example-Based Super-Resolution. In Proceedings of the 2013 IEEE International Conference on Computer Vision, Sydney, Australia, 1-8 December 2013; pp. 1920-1927.

45. Kawakami, R.; Matsushita, Y.; Wright, J.; Ben-Ezra, M.; Tai, Y.W.; Ikeuchi, K. High-resolution hyperspectral imaging via matrix factorization. In Proceedings of the CVPR 2011, Colorado Springs, CO, USA, 20-25 June 2011; pp. 2329-2336. [CrossRef]

46. Larsson, G.; Maire, M.; Shakhnarovich, G. Learning representations for automatic colorization. In Proceedings of the Lecture Notes in Computer Science (Including Subseries Lecture Notes in Artificial Intelligence and Lecture Notes in Bioinformatics); Springer: Berlin/Heidelberg, Germany, 2016; Volume 9908, pp. 577-593.

47. Deshpande, A.; Rock, J.; Forsyth, D. Learning large-scale automatic image colorization. In Proceedings of the IEEE International Conference on Computer Vision, Santiago, Chile, 7-13 December 2015.

48. Morimoto, Y.; Taguchi, Y.; Naemura, T. Automatic colorization of grayscale images using multiple images on the web. In Proceedings of the SIGGRAPH 2009: Talks, New Orleans, LA, USA, 3-7 August 2009. [CrossRef]

49. Chia, A.Y.S.; Zhuo, S.; Tan, P.; Gupta, R.K.; Cho, S.Y.; Tai, Y.W.; Lin, S. Semantic Colorization with Internet Images. ACM Trans. Graph. 2011, 30, 1-8. [CrossRef]

50. Gupta, R.K.; Chia, A.Y.S.; Rajan, D.; Ng, E.S.; Zhiyong, H. Image Colorization using Similar Images; ACM Press: New York, NY, USA, 2012; pp. 369-378.

51. Ironi, R.; Cohen-Or, D.; Lischinski, D. Colorization by Example. Render. Tech. 2005, 29, 201-210.

52. Bugeau, A.; Ta, V.T.; Papadakis, N. Variational exemplar-based image colorization. IEEE Trans. Image Process. 2014, $23,298-307$. [CrossRef]

53. Román, M.O.; Wang, Z.; Shrestha, R.; Yao, T.; Kalb, V. Black Marble User Guide Version 1.0; NASA: Washington, DC, USA, 2019.

54. Wang, P.; Huang, C.; Brown de Colstoun, E.C.; Tilton, J.C.; Tan, B. Global Human Built-Up And Settlement Extent (HBASE) Dataset From Landsat; NASA Socioeconomic Data and Applications Center (SEDAC): Palisades, NJ, USA, 2017. 
55. ArcGIS Desktop I Desktop GIS Software Suite-Esri. Available online: https://www.esri.com/en-us/arcgis/products/arcgisdesktop/overview (accessed on 2 November 2021).

56. GitHub. Elastic Map. Available online: https://github.com/Mirkes/ElMap (accessed on 11 April 2020).

57. Truong, T.-D.; Nguyen, V.-T.; Tran, M.-T. Lightweight Deep Convolutional Network for Tiny Object Recognition. In Proceedings of the 7th International Conference on Pattern Recognition Applications and Methods 2018, Funchal, Portugal, 16-18 January 2018 [CrossRef]

58. MATLAB-MathWorks-MATLAB \& Simulink. Available online: https://www.mathworks.com/products/matlab.html (accessed on 11 September 2021).

59. Train Deep Learning Neural Network-MATLAB Train Network. Available online: https://www.mathworks.com/help/ deeplearning/ref/trainnetwork.html (accessed on 11 September 2021).

60. JASP. Available online: https:/ /jasp-stats.org/ (accessed on 20 September 2021).

61. A New Approach to Identify On-Ground Lamp Types from Night-Time ISS Images. Available online: https:/ / eartharxiv.org/ repository/view/2684/ (accessed on 19 September 2021). 